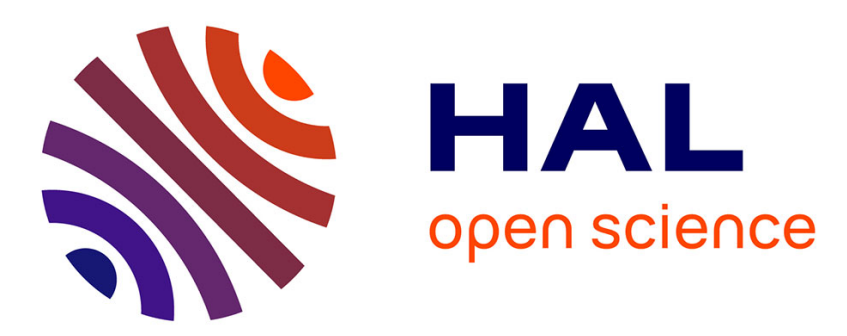

\title{
Pose and covariance matrix propagation issues in cooperative localization with LiDAR perception
}

Elwan Héry, Philippe Xu, Philippe Bonnifait

\section{To cite this version:}

Elwan Héry, Philippe $\mathrm{Xu}$, Philippe Bonnifait. Pose and covariance matrix propagation issues in cooperative localization with LiDAR perception. 30th IEEE Intelligent Vehicles Symposium (IV 2019), Jun 2019, Paris, France. pp.1219-1224. hal-02407423

\section{HAL Id: hal-02407423 \\ https://hal.science/hal-02407423}

Submitted on 12 Dec 2019

HAL is a multi-disciplinary open access archive for the deposit and dissemination of scientific research documents, whether they are published or not. The documents may come from teaching and research institutions in France or abroad, or from public or private research centers.
L'archive ouverte pluridisciplinaire $\mathbf{H A L}$, est destinée au dépôt et à la diffusion de documents scientifiques de niveau recherche, publiés ou non, émanant des établissements d'enseignement et de recherche français ou étrangers, des laboratoires publics ou privés. 


\title{
Pose and covariance matrix propagation issues in cooperative localization with LiDAR perception
}

\author{
Elwan Héry ${ }^{1}$, Philippe $\mathrm{Xu}^{1}$ and Philippe Bonnifait ${ }^{1}$
}

\begin{abstract}
This work describes a cooperative pose estimation solution where several vehicles can perceive each other and share a geometrical model of their shape via wireless communication. We describe two formulations of the cooperation. In one case, a vehicle estimates its global pose from the one of a neighbor vehicle by localizing it in its body frame. In the other case, a vehicle uses its own pose and its perception to help localizing another one. An iterative minimization approach is used to compute the relative pose between the two vehicles by using a LiDAR-based perception method and a shared polygonal geometric model of the vehicles. This study shows how to obtain an observation of the pose of one vehicle given the perception and the pose communicated by another one without any filtering to properly characterize the cooperative problem independently of any other sensor. Accuracy and consistency of the proposed approaches are evaluated on real data from on-road experiments. It is shown that this kind of strategy for cooperative pose estimation can be accurate. We also analyze the advantages and drawbacks of the two approaches on a simple case study.
\end{abstract}

\section{INTRODUCTION}

Localization is a key feature for autonomous driving, in particular when vehicles cooperate in complex situations like lane merging on a motorway for instance [21]. The cooperation between vehicles can improve localization and this can be achieved through the use of new communication standards such as CAM (Cooperative Awareness Message) [8] to receive the pose of other vehicles and CPM (Cooperative Perception Message) to share perception information. Moreover, cooperative localization is a well-known issue in mobile robotics [14], [16], [17], [12]. Perception is often described as bearing and range, sometimes with relative orientation. It is then essential to use multiple perceived robots to localize one robot of the fleet. It has also been shown that vehicles can improve their own pose estimates thanks to perception information and pose estimates of neighboring vehicles [11], [9]. However, these works often use simulated data, the perception problem is then not always perfectly represented.

A typical scenario of cooperative localization aided by perception is cooperative navigation through a local GNSS denied environment. For example, when a platoon of vehicles goes under a bridge, the vehicles of the platoon lose their GNSS signals one after the others, Fig. 1. The vehicles that are not under the bridge are able to help the vehicles which have lost their absolute position measurements.

\footnotetext{
${ }^{1}$ The authors are with Sorbonne universités, Université de technologie de Compiègne, CNRS, Heudiasyc UMR 7253, 60203 Compiègne Cedex, France firstname.surnamedhds.utc.fr
}
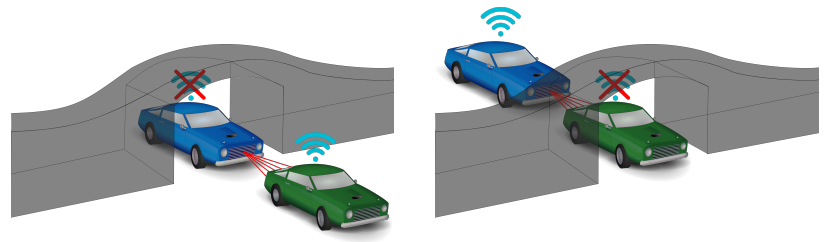

(a) First formulation.

(b) Second formulation.

Fig. 1. Platoon of two vehicles losing the GNSS signals while driving under a bridge.

In order for a vehicle to make use of the pose estimate of another one, it needs to be able to estimate the relative pose between them. Camera based vehicle detection has been extensively studied in the literature in the past decades. Recent deep learning based detectors reached relatively high accuracy for relative pose estimation in the image space [4]. However, monocular vision based methods often do not provide accurate metric pose estimates. 3D point clouds given by stereovision or LiDAR can be used to obtain metric pose estimates. By fitting the points with a model, e.g., Lshape fitting [22] or more general 3D model fitting [5], the relative pose w.r.t. a detected vehicle can be estimated. To compute this relative pose, the Iterative Closest Point (ICP) method, often used for scan matching [3], can be applied [10].

For multiple cooperative vehicles to make the most of exchanged pose estimates within a data fusion framework, each vehicle not only needs to be able to provide a pose estimate but also needs to properly quantify the uncertainty associated with it. When using a classical Gaussian assumption, it is done through the estimation of the covariance matrix. Therefore, the ability to have a consistent estimation of the covariance matrix associated to the perception [1], [2], [15] becomes of significant importance.

In the literature, cooperative localization problems using data fusion often describe the state of one vehicle with the pose and the velocity of this vehicle and of its neighbor and use the relative measurements as observations. In this work, we show how a vehicle can propagate the pose estimate and the associated uncertainty of a neighboring vehicle by using the LiDAR based perception approach combined with the exchange of geometrical models of the vehicles proposed in [10]. This relative localization method can be used even when only the back of the vehicle is in the field of view of the LiDAR. Other more classical methods such as L-shape fitting need two sides of the vehicle. The contribution of 


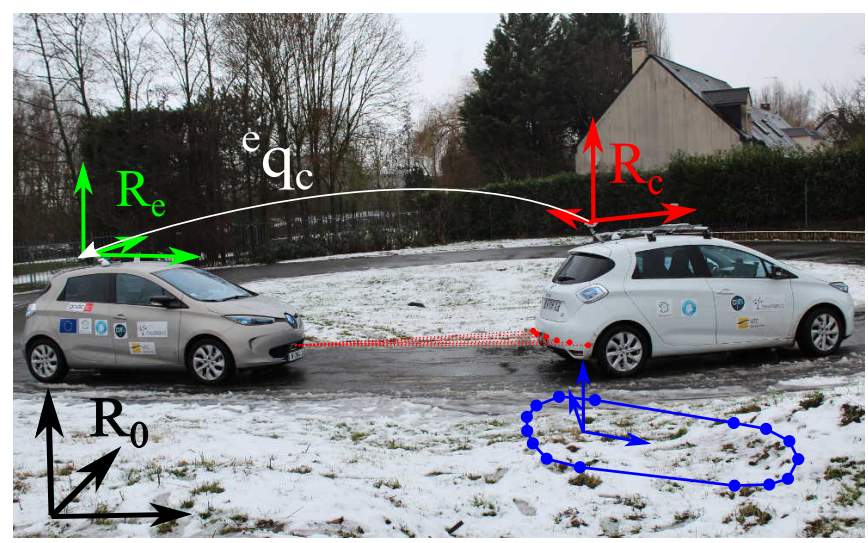

Fig. 2. Two experimental vehicles equipped with GNSS receivers and LiDAR sensors on a test road at Compiègne, France. The LiDAR points are shown in red and the polygonal model is in blue. This figure shows the relative pose ${ }^{e} q_{c}$ of the mobile frame $R_{c}$ of the cooperating vehicle (white car) in the frame $R_{e}$ of the ego-vehicle (gray car). $R_{0}$ is a local working frame in which the vehicles need to be localized for their navigation tasks.

this paper is to show the accuracy and consistency of the cooperative pose estimation using real LiDAR perception, without any filtering, to properly characterized this raw observation computed from one epoch only without the use of other sensors, e.g. GNSS or odometry observations. The results of the proposed methods can be seen as observations which can be used in the update of any filtering algorithm, e.g., Extended Kalman Filter or covariance intersection filter. This approach has the advantage of only requiring another vehicle to estimate the position and the orientation. In the case of multiple surrounding cars, each vehicle can be seen as a different observation.

We consider two formulations of the cooperative localization problem. In the first one, the ego-vehicle perceives a cooperating vehicle and uses the pose estimate of the latter to estimate its own pose. In the second formulation, we consider that it is the ego-vehicle that is perceived by the cooperating vehicle which can directly help estimate the pose of the egovehicle. The relative pose between the vehicles is estimated by minimizing the distances between LiDAR scans and a polygonal model of the shape of the vehicles.

Both accuracy and consistency of the localization resulting from the two formulations are evaluated and compared using real data recorded from two communicating experimental vehicles in the city of Compiègne, France (see Fig. 2).

The cooperative localization problem is first introduced in section II, where we present the pose and the covariance propagation. The relative localization process based on an iterative minimization method is explained in section III. Experimental results are studied and analyzed in section IV. We finally analyze the advantages and drawbacks of the two approaches on a simple case study.

\section{Problem statement}

As stated in the previous section, the aim of this work is to estimate the pose of a given vehicle from the localization information of another communicating vehicle via perception information. Throughout this work, we consider that the vehicles are equipped with a LiDAR capable of detecting surrounding vehicles. In order to be able to estimate an accurate relative pose between two vehicles, in particular relative orientation, we also suppose that they can exchange a model of their geometrical shape as illustrated in Fig. (2).

Using a 2D formulation is often sufficient in vehicle localization problems as many road environments are locally planar. Therefore, the pose of a vehicle is defined by its 2D coordinates and its heading : $q=[x y \theta]^{T}$. The data coming from the LiDAR sensor is considered as a set of twodimensional points $P=\left\{p_{i}\right\}_{i=1, \ldots, N}$. We suppose that this set of points corresponds to a detected vehicle. The detection task itself is out of the scope of this paper, many LiDAR clustering algorithms can be found in the literature [6]. The shape of the vehicle is also given in $2 \mathrm{D}$ by a polygonal model representing the outer hull from a bird's-eye view. A shape model is represented as a set of consecutive points $M=\left(m_{1}, \ldots, m_{K}\right)$.

The considered 2D geometrical shapes are typically defined by the bumpers of the vehicles which are situated at the most outer place. For safety reasons in case of collision, the height of the bumpers is almost the same for the majority of vehicles. Therefore, placing a LiDAR layer at this same height provides a perception that is consistent with the considered models. This assumption may not be respected in some cases, e.g., for trucks or non-planar roads. In this situation, a multi-layer 3D LiDAR may be used to enhance the detection of the 2D hull of a detected vehicle. The method proposed in this paper can be generalized to this 3D case.

Let us consider a first approach in which the ego-vehicle aims at estimating its own pose $q_{e}$ using the pose $q_{c}$ of a cooperating vehicle as a deported antenna with a lever arm corresponding to their relative pose ${ }^{e} q_{c}$ (expressed in the mobile frame of the ego-vehicle). This relative pose is estimated using perception. Here, the goal is to find an estimate $\hat{q}_{e}$ in a fixed working frame along with its associated covariance matrix $\widehat{\Sigma}_{e}$ using the estimates $\hat{q}_{c}, \widehat{\Sigma}_{c},{ }^{e} \hat{q}_{c}$ and ${ }^{e} \widehat{\Sigma}_{c}$.

Let $R(\theta)$ be the rotation matrix defined as

$$
R(\theta)=\left[\begin{array}{ccc}
\cos (\theta) & -\sin (\theta) & 0 \\
\sin (\theta) & \cos (\theta) & 0 \\
0 & 0 & 1
\end{array}\right]
$$

Suppose that the relative pose is estimated from the egovehicle point of view. In this first formulation, the pose of the ego-vehicle is computed with the following equation:

$$
\begin{aligned}
q_{e} & =q_{c}-R\left(\theta_{e}\right){ }^{e} q_{c} \\
& =\left[\begin{array}{c}
x_{c}-{ }^{e} x_{c} \cdot \cos \left(\theta_{e}\right)+{ }^{e} y_{c} \cdot \sin \left(\theta_{e}\right) \\
y_{c}-{ }^{e} x_{c} \cdot \sin \left(\theta_{e}\right)-{ }^{e} y_{c} \cdot \cos \left(\theta_{e}\right) \\
\theta_{c}-{ }^{e} \theta_{c}
\end{array}\right] \\
& =\left[\begin{array}{c}
x_{c}-{ }^{e} x_{c} \cdot \cos \left(\theta_{c}-{ }^{e} \theta_{c}\right)+{ }^{e} y_{c} \cdot \sin \left(\theta_{c}-{ }^{e} \theta_{c}\right) \\
y_{c}-{ }^{e} x_{c} \cdot \sin \left(\theta_{c}-{ }^{e} \theta_{c}\right)-{ }^{e} y_{c} \cdot \cos \left(\theta_{c}-{ }^{e} \theta_{c}\right) \\
\theta_{c}-{ }^{e} \theta_{c}
\end{array}\right] .
\end{aligned}
$$

Since the relation $q_{e}=f_{1}\left(q_{c},{ }^{e} q_{c}\right)$ is not linear, a first order approximation is used to estimate $\Sigma_{e}$ from $\Sigma_{c}$ and ${ }^{e} \Sigma_{c}$, as 
presented by [7], [18]:

$$
\Sigma_{e} \approx \frac{\partial f_{1}}{\partial q_{c}} \Sigma_{c}{\frac{\partial f_{1}}{\partial q_{c}}}^{T}+\frac{\partial f_{1}}{\partial^{e} q_{c}} \Sigma_{c}{\frac{\partial f_{1}}{\partial^{e} q_{c}}}^{T}
$$

where the Jacobian matrices of $f_{1}, \frac{\partial f_{1}}{\partial q_{c}}$ and $\frac{\partial f_{1}}{\partial^{e} q_{c}}$ are defined as follows:

$$
\begin{gathered}
\frac{\partial f_{1}}{\partial q_{c}}=\left[\begin{array}{ccc}
1 & 0 & { }^{e} x_{c} s_{1}+{ }^{e} y_{c} c_{1} \\
0 & 1 & -{ }^{e} x_{c} c_{1}+{ }^{e} y_{c} s_{1} \\
0 & 0 & 1
\end{array}\right], \\
\frac{\partial f_{1}}{\partial^{e} q_{c}}=\left[\begin{array}{ccc}
-c_{1} & s_{1} & -{ }^{e} x_{c} s_{1}-{ }^{e} y_{c} c_{1} \\
-s_{1} & -c_{1} & { }^{e} x_{c} c_{1}-{ }^{e} y_{c} s_{1} \\
0 & 0 & -1
\end{array}\right],
\end{gathered}
$$

with $s_{1}=\sin \left(\theta_{c}-{ }^{e} \theta_{c}\right)$ and $c_{1}=\cos \left(\theta_{c}-{ }^{e} \theta_{c}\right)$.

Another possible solution is to have the relative pose computed from the cooperating vehicle $\left({ }^{c} q_{e}\right)$. The pose of the ego-vehicle is then estimated as follows:

$$
\begin{aligned}
q_{e} & =q_{c}+R\left(\theta_{c}\right){ }^{c} q_{e} \\
& =\left[\begin{array}{c}
x_{c}+{ }^{c} x_{e} \cdot \cos \left(\theta_{c}\right)+{ }^{c} y_{e} \cdot \sin \left(\theta_{c}\right) \\
y_{c}+{ }^{c} x_{e} \cdot \sin \left(\theta_{c}\right)-{ }^{c} y_{e} \cdot \cos \left(\theta_{c}\right) \\
\theta_{c}+{ }^{c} \theta_{e}
\end{array}\right] .
\end{aligned}
$$

Notice that using this formulation $q_{e}=f_{2}\left(q_{c}{ }^{c} q_{e}\right)$, the position coordinates $\left(x_{e}, y_{e}\right)$ become independent of the relative orientation ${ }^{c} \theta_{e}$. This implies that the position estimation becomes more robust to inaccurate estimation of the relative orientation. Similarly to the previous case, the covariance matrix is estimated using Jacobian matrices

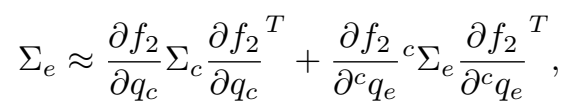

where

$$
\begin{gathered}
\frac{\partial f_{2}}{\partial q_{c}}=\left[\begin{array}{ccc}
1 & 0 & 0 \\
0 & 1 & 0 \\
0 & 0 & 1
\end{array}\right], \\
\frac{\partial f_{2}}{\partial^{c} q_{e}}=\left[\begin{array}{ccc}
c_{2} & -s_{2} & -{ }^{c} x_{e} s_{2}-{ }^{c} y_{e} c_{2} \\
s_{2} & c_{2} & { }^{c} x_{e} c_{2}-{ }^{c} y_{e} s_{2} \\
0 & 0 & 1
\end{array}\right],
\end{gathered}
$$

with $s_{2}=\sin \left(\theta_{c}\right)$ and $c_{2}=\cos \left(\theta_{c}\right)$.

\section{RELATIVE LOCALIZATION}

\section{A. LiDAR based perception}

The Iterative Closest Point (ICP) algorithm is often used to match two LiDAR scans in order to obtain the relative pose between them. In our case, we aim at matching two sets of points but with one that corresponds to a LiDAR scan and another that represents the polygonal shape model of a vehicle. However, these two sets do not come from the same sensor as in classical ICP resulting in a disparity in terms of the number of points. Therefore, we use a variant of the ICP algorithm that uses line segments to represent the model points, [10], [3], [13]. The principle of the ICP is to have an iterative process that alternates a matching step with a minimization one. This relative localization problem using LiDAR measurements and a polygonal model has been

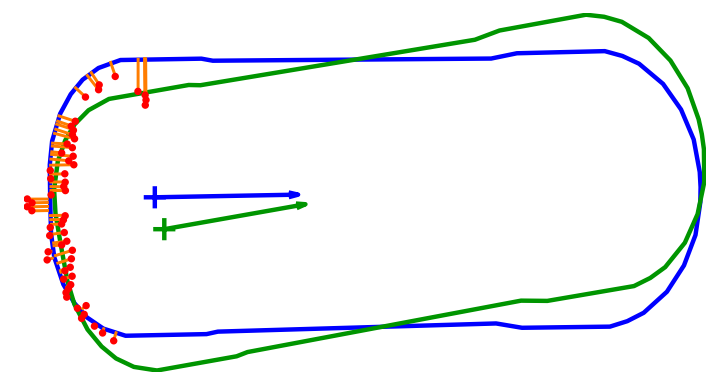

Fig. 3. Distances to minimize in orange between the LiDAR point in red and the model in blue. The initial pose communicated by the cooperating vehicle is shown in blue and the final pose is in green.

described and compared to other approaches in [10]. In practice, only one cluster of points of the scan is matched with the model. This clustering step and the matching of the different clusters with the models of different vehicles in the case of a multiple vehicles scenario is not studied in this paper. A consistency test between the computed relative pose using this algorithm and the initial relative pose can be computed to check if the matching between the cluster and the vehicle model is correct.

This method provides an estimate of the relative pose which minimizes the distances between the LiDAR points, in red, and the model, in blue in Fig. 3. These distances are shown in orange in Fig. 3.

Once each LiDAR point is matched with one segment of the polygonal model, the minimization problem can be written as follows:

$$
\min _{q} E(q ; P, M)=\min _{q} \sum_{i=1}^{N} d\left(q ; p_{i}, M\right)^{2},
$$

where $d\left(q ; p_{i}, M\right)$ is the distance between the point $p_{i}$ and the model $M$ when the transformation associated to the pose $q$ is applied to the LiDAR scan $P$.

The authors of [13] showed that this minimization problem can be simplified as a quadratic convex problem with the following form:

$$
E(\Delta q ; P, M)=\|A \Delta q-b\|^{2} .
$$

This problem can be solved using the pseudo-inverse of the matrix $A$ :

$$
\Delta \hat{q}=\operatorname{pinv}(A) b .
$$

Once the pose is found, the covariance matrix can be computed, as described in [1]:

$$
\Delta \widehat{\Sigma}=2 \frac{E(\Delta \hat{q})}{N-D}\left(\frac{\partial^{2} E}{\partial q^{2}}(\Delta \hat{q})\right)^{-1}=\frac{E(\Delta \hat{q})}{N-D}\left(A^{T} A\right)^{-1},
$$

where $D$ is the dimension of $\Delta q$, i.e., $D=3$.

It is then possible to estimate the relative pose ${ }^{e} \hat{q}_{c, k}$ and its associated covariance matrix for this iteration $k$ using the previous iteration ${ }^{e} \hat{q}_{c, k-1}$ and the pose $\Delta \hat{q}_{k}$ obtain with the minimization:

$$
{ }^{e} \hat{q}_{c, k}={ }^{e} \hat{q}_{c, k-1}-R\left({ }^{e} \theta_{c, k-1}\right) \Delta \hat{q}_{k},
$$




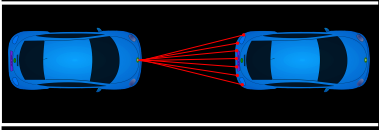

(a) C-shape.

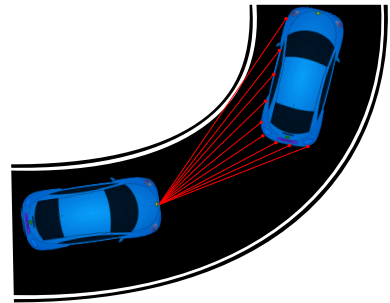

(b) L-shape.
Fig. 4. LiDAR detections in a straight line and a curved line.

$$
{ }^{e} \hat{\Sigma}_{c, k}=R\left({ }^{e} \theta_{c, k}\right) \Delta \widehat{\Sigma}_{k} R\left({ }^{e} \theta_{c, k}\right)^{T},
$$

where $R\left({ }^{e} \theta_{c, k}\right)$ is the rotation matrix with the angle ${ }^{e} \theta_{c, k}$.

Once the ICP has converged, the pose ${ }^{e} \hat{q}_{c, k}$ and the covariance matrix ${ }^{e} \hat{\Sigma}_{c, k}$ of the last iteration gives a good estimate of the relative pose and of its covariance matrix.

\section{B. Propagation accuracy and consistency}

1) LiDAR detections: In real driving scenarios the LiDAR perception does not always provide accurate relative localization. When only the back of the vehicle is in the field of view of the LiDAR a C-shape perception can be seen, Fig. 4a. This shape creates a rotation invariance during the execution of the ICP algorithm which leads to larger relative heading and lateral errors. When the back and one side of the vehicle are in the field of view of the LiDAR, an L-shape is visible, Fig. $4 \mathrm{~b}$. The angle ambiguity disappears and the relative orientation becomes accurate.

Moreover, the position obtained by the first formulation depends on the relative orientation which is not the case for the second formulation. This is visible when the relative heading is badly estimated in Fig. 5b when a C-shape is visible and when the first formulation is used. In Fig. 5d, when a C-shape is detected and when the second formulation is used, even if the relative heading is not accurate, the obtained position is not impacted.

2) Non linearity issues during covariance matrix propagation: The inconsistency of the first formulation is therefore only due to the highly non-linear mathematical expressions in Eq. (2). Fig. 6 illustrates through Monte Carlo simulations the empirical distribution of the estimated pose of the egovehicle when the communicated pose (in black) and the relative pose have Gaussian distributions. One can see that the results are actually following banana-shaped distributions [20], [19] which are badly approximated by Gaussian distributions when they are large. The first formulation (in blue) gives a distribution less Gaussian than the second one (in red) because of the dependency of the relative orientation error, see Eq. (3). Both formulations are dependent on the orientation error of the communicated pose, as shown by Eq. (3) and (6). Therefore, both formulations give bananashaped distributions. The propagation of the uncertainty through the non-linear transformation is an approximation.

With the second formulation, the cooperating vehicle computes the pose of the ego-vehicle for it with a good un- certainty estimate. Therefore, the ego-vehicle pose estimate is entirely dependent on the cooperating vehicle. This is acceptable if the cars are in a trustworthy network but it can lead to trust issues in other real case scenarios. The first formulation does not make this assumption. Indeed, the relative pose is computed with the perception sensor of the ego-vehicle. Misleading information sent by the cooperating vehicle such as erroneous pose estimate or wrong mode can be detected by using the error computed from the minimization step as a trust indicator. Indeed, if the cooperating vehicle does not send an accurate pose or model, it will not be coherent with the estimated pose of the ego-vehicle. This can be detected by its perception leading to a large value of the cost function.

\section{EXPERIMENTAL RESULTS}

\section{A. Experimental platform}

The dataset used for this experimentation was recorded on a platooning scenario where two Renault Zoe cars were driving on a test road composed of two roundabouts and a straight lane between them, as pictured in Fig. 2. Nine laps were completed in this test track for a total of $2.82 \mathrm{~km}$ in 10 minutes and 7,489 LiDAR scans. The inter-distance between the poses of the two vehicles was between $6.2 \mathrm{~m}$ and $18.8 \mathrm{~m}$. This inter-distance was around $8 \mathrm{~m}$ in the roundabouts and $13 \mathrm{~m}$ in the straight lane. Each vehicle was equipped with a Novatel's SPAN-CPT, an IMU with a GNSS receiver using RTK corrections for the ground truth. No tracking or data fusion was implemented in this work. The frequency of the ground truth being high compared to the speed of the vehicle, the temporal error was supposed to be negligible compared to the spatial error. The following vehicle was also equipped with a four-layer SICK LDMRS LiDAR, but only one layer was used to compute the relative pose. The 2D geometric model of the Renault Zoe car has been manually built from a blueprint. The ground truth having an uncertainty in the order of one decimeter, its covariance matrix was used to compute the consistency. Since the error of the ground truth is not negligible compared to the LiDAR measurement, small errors should be interpreted carefully.

To evaluate the consistency, we test the following condition:

$$
\left(\hat{q}_{e}-q_{r e f}\right)^{T}\left(\hat{\Sigma}_{e}+\Sigma_{r e f}\right)^{-1}\left(\hat{q}_{e}-q_{r e f}\right) \leq \chi_{3 ; 0.05}^{2},
$$

where $\chi_{3 ; 0.05}^{2}=7.815$ is the quantile of a $\chi^{2}$ distribution with 3 degrees of freedom and a confidence level of $95 \%$. $q_{r e f}$ and $\Sigma_{r e f}$ are the pose and the covariance of the ground truth. In practice, it means that Eq. (16) has to be verified for at least for $95 \%$ of the samples.

Table I reports the results of the experiments. We decomposed the horizontal error $e_{h}$ into a longitudinal $e_{x}$ and lateral $e_{y}$ components in the mobile frame of the ground truth for a better analysis.

\section{B. First formulation}

The ego-vehicle computes its pose from its own perception sensor given the pose and the model received by the cooperating vehicle (see Fig. 5 (a-b)). 


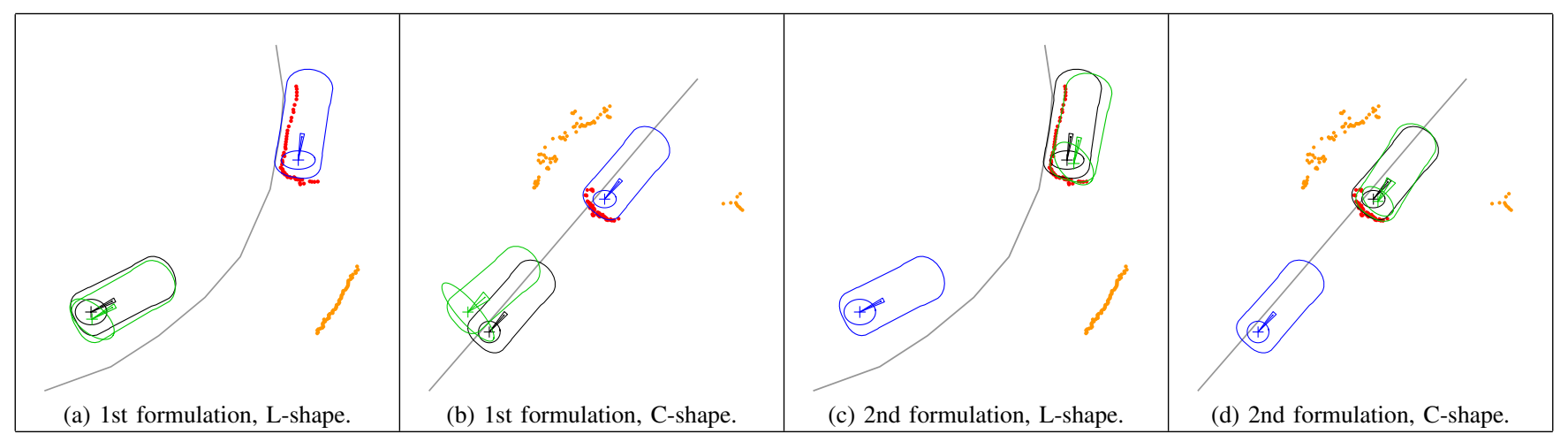

Fig. 5. Estimated pose of the ego-vehicle, in green, computed from the pose of the cooperating vehicle, in blue, and the LiDAR points of the cluster, in red. This pose is compared to the ground truth in black. The gray polyline is the center of the road. For each pose, the model and the uncertainty ellipse and the uncertainty cone are shown in the same color.

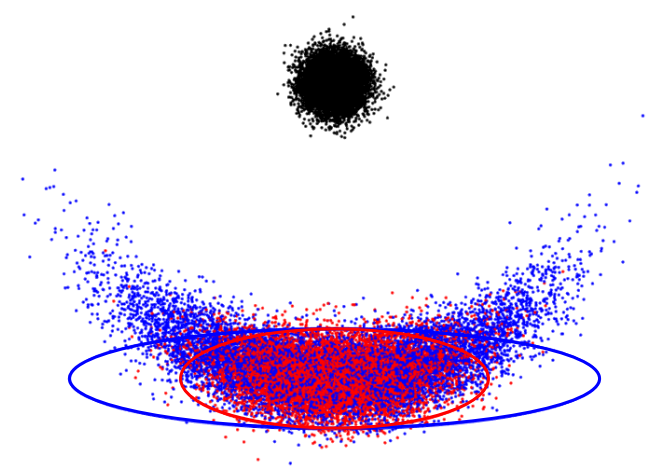

Fig. 6. Banana shape distribution of the estimated pose and its $95 \%$ confidence ellipse approximation, with the first formulation in blue and with the second formulation in red, from the communicated pose in black.

TABLE I

MEAN ERRor $\left[e_{x} e_{y} e_{\theta}\right]^{T}$ IN THE FRAME OF THE GROUND TRUTH, MEAN HORIZONTAL ERROR $e_{h}$ AND CONSISTENCY RATE $C$ OF THE TWO FORMULATIONS FOR THE COMPLETE SCENARIO, FOR THE L-SHAPE PERCEPTIONS ONLY AND FOR THE C-SHAPE PERCEPTIONS ONLY.

\begin{tabular}{|c|c|c|c|c|c|c|}
\cline { 2 - 7 } \multicolumn{1}{c|}{} & \multicolumn{3}{c|}{ 1st formulation } & \multicolumn{3}{c|}{ 2nd formulation } \\
\hline shape & all & C & L & all & C & L \\
\hline$e_{x}(\mathrm{~m})$ & 0.27 & 0.35 & 0.13 & 0.11 & 0.11 & 0.09 \\
\hline$e_{y}(\mathrm{~m})$ & 1.16 & 1.72 & 0.22 & 0.36 & 0.41 & 0.27 \\
\hline$e_{h}(\mathrm{~m})$ & 1.24 & 1.82 & 0.27 & 0.39 & 0.44 & 0.29 \\
\hline$e_{\theta}\left({ }^{\circ}\right)$ & 6.92 & 8.55 & 4.20 & 6.92 & 8.55 & 4.20 \\
\hline$C(\%)$ & 91.5 & 86.9 & 99.2 & 98.0 & 97.2 & 99.3 \\
\hline
\end{tabular}

This first formulation gives an accuracy of $1.24 \mathrm{~m}$ for the position and $6.92^{\circ}$ for the orientation. This is not accurate enough for autonomous driving. The consistency rate is 91.5\% and so the pose estimate is not consistent.

Two different perceptions of the cooperating vehicle exist in this platooning scenario: an L-shape, when the back and one side of the vehicle are in the field of view of the LiDAR and a C-shape, when only the back is in the field of view.

1) L-shape: L-shape can be seen by the LiDAR when both vehicles are driving on the roundabout, Fig. 5-a. This perception gives a good accuracy, $0.27 \mathrm{~m}$ for the position and $4.20^{\circ}$ for the orientation. The covariance also becomes a bit pessimistic with a $99.2 \%$ consistency rate.
2) C-shape: C-shape can be seen by the LiDAR when both vehicles are driving on the straight lane, Fig. 5-b. The back of the car being curved, a rotation invariance appears. The LiDAR points are free to move along the back of the model without changing the error. From Eq. (2), one can see that the estimated position of the ego-vehicle is dependent on the relative orientation. We obtain an accuracy of $1.82 \mathrm{~m}$ for the position and $8.55^{\circ}$ for the orientation. The covariance is less consistent for this kind of perception (86.9\%).

As one might expect, the lateral error, in the frame of the ground truth, is the largest part of the error, as shown by the uncertainty ellipse in Fig. 5b. This error is of $1.72 \mathrm{~m}$ compared to $0.35 \mathrm{~m}$ for the longitudinal error.

\section{Second formulation}

The cooperating vehicle computes the pose of the egovehicle with its pose and its perception sensor, Fig. 5 (cd). This second formulation of the cooperative localization problem gives a better accuracy, with $0.39 \mathrm{~m}$ for the position and $6.92^{\circ}$ for the orientation. It also has a very good consistency rate of $98.0 \%$. Indeed from Eq. (6), one can see that the estimated position of the ego-vehicle does not depend on the relative orientation anymore. One can also see that the orientation error does not change from one formulation to the other.

1) L-shape: As for the first formulation, the pose found for this perception is very accurate with a mean error of $0.29 \mathrm{~m}$ for the position and $4.20^{\circ}$ for the orientation and also a bit too pessimistic (consistency rate of $99.3 \%$ ).

2) $C$-shape: This perception is much more accurate and provides consistent information for this formulation. Indeed, the position is independent of the relative orientation. The mean error is $0.44 \mathrm{~m}$ for the position and $8.55^{\circ}$ for the orientation and the consistency is $97.2 \%$ which is excellent.

\section{Analysis}

Based on these results, we can conclude that it is more accurate and more consistent to use the perception of the vehicle sending its pose than computing the pose of the ego-vehicle from its own perception. The accuracy of the 
second formulation is better because the position estimation is independent of the relative orientation uncertainty. The inconsistency of the global estimate possibly comes from an underestimation of the covariance matrix of the relative pose $e \widehat{\Sigma}_{c}$. However, as the second formulation is consistent, it is reasonable to assume that ${ }^{e} \widehat{\Sigma}_{c}$ is actually consistent.

In conclusion, if two cooperative vehicles are driving together the vehicle with the most accurate localization should drive behind the other. Nevertheless, even if the second formulation gives better results, both formulations can be necessary in some scenarios, for example when only one vehicle is able to perceive the other.

\section{CONCLUSION}

This work has presented two formulations of a cooperative localization problem using the pose and shape of another vehicle. To compute the relative pose from the LiDAR points, an iterative minimization problem has been applied with points to lines matching. This led to a relatively consistent estimation of the relative pose. Two formulations have been tested experimentally and the accuracy and consistency have been evaluated in different situations. On curved roads where L-shapes can be perceived, both formulations gave good results with an accuracy that is of the order of magnitude of the decimeter. However, on straight lanes where the perception leads to $\mathrm{C}$-shapes, the second formulation is more consistent and more accurate.

In future work, this cooperative localization method will be used with data fusion to improve the accuracy while keeping a good integrity. Different challenging scenarios will be tested, e.g., multiple vehicles in long tunnels or having only one vehicle accurately localized in a platoon. The cooperative method proposed in this paper can also be extended when two vehicles are perceiving a same feature of the environment. One vehicle can communicate its observation and the other one can localize itself using this shared observation.

\section{ACKNOWLEDGMENT}

This work was carried out in the framework of the Equipex ROBOTEX (ANR-10- EQPX-44-01) and Labex MS2T (ANR-11-IDEX-000402) projects. It was also carried out within SIVALab, a shared laboratory between Renault and Heudiasyc UMR UTC/CNRS.

\section{REFERENCES}

[1] O. Bengtsson and A.-J. Baerveldt. Robot localization based on scanmatching-estimating the covariance matrix for the IDC algorithm. Robotics and Autonomous Systems, 44(1):29-40, July 2003.

[2] A. Censi. An accurate closed-form estimate of ICP's covariance. In Proceedings IEEE International Conference on Robotics and Automation, pages 3167-3172, April 2007.

[3] A. Censi. An ICP variant using a point-to-line metric. In IEEE International Conference on Robotics and Automation, pages 19-25, May 2008.

[4] T. Chateau, F. Chabot, C. Teulière, M. Chaouch, and J. Rabarisoa. Deep MANTA: A Coarse-to-Fine Many-Task Network for Joint 2d and $3 \mathrm{~d}$ Vehicle Analysis from Monocular Image. In IEEE Conference on Computer Vision and Pattern Recognition (CVPR), Honolulu, USA, 2017.
[5] Max Coenen, Franz Rottensteiner, and Christian Heipke. Detection and $3 \mathrm{~d}$ modelling of vehicles from terrestrial stereo image pairs. International Archives of the Photogrammetry, Remote Sensing and Spatial Information Sciences - ISPRS Archives 42 (2017), Nr. 1W1, 42(1W1):505-512, 2017.

[6] B. Douillard, J. Underwood, N. Kuntz, V. Vlaskine, A. Quadros, P. Morton, and A. Frenkel. On the segmentation of 3d LIDAR point clouds. In IEEE International Conference on Robotics and Automation, pages 2798-2805, May 2011.

[7] H. F. Durrant-Whyte. Uncertain geometry in robotics. IEEE Journal on Robotics and Automation, 4(1):23-31, February 1988.

[8] ETSI. Intelligent transport systems (ITS); Vehicular communications; Basic set of applications; Part 2: specification of cooperative awareness basic service. Technical Report ETSI EN 302 637-2 v1.3.1, Sep. 2014.

[9] E. Héry, $\mathrm{Ph} . \mathrm{Xu}$, and $\mathrm{Ph}$. Bonnifait. Along-track localization for cooperative autonomous vehicles. In IEEE Intelligent Vehicles Symposium, pages 11-14, Redondo Beach CA, USA, jun 2017.

[10] E. Héry, $\mathrm{Ph} . \mathrm{Xu}$, and $\mathrm{Ph}$. Bonnifait. LiDAR based relative pose and covariance estimation for communicating vehicles exchanging a polygonal model of their shape. 10th Workshop on Planning, Perception and Navigation for Intelligent Vehicles, october 2018.

[11] N. Karam, F. Chausse, R. Aufrere, and R. Chapuis. Localization of a Group of Communicating Vehicles by State Exchange. In IEEE/RSJ International Conference on Intelligent Robots and Systems, pages 519-524, October 2006.

[12] R. Kurazume, S. Nagata, and S. Hirose. Cooperative positioning with multiple robots. In Proceedings of the 1994 IEEE International Conference on Robotics and Automation, pages 1250-1257 vol.2, May 1994.

[13] K.-L. Low. Linear Least-Squares Optimization for Point-to-Plane ICP Surface Registration. Technical Report TR04-004, Department of Computer Science University of North Carolina at Chapel Hill, February 2004.

[14] A. Martinelli, F. Pont, and R. Siegwart. Multi-Robot Localization Using Relative Observations. In Proceedings of the IEEE International Conference on Robotics and Automation, pages 2797-2802, April 2005.

[15] S. M. Prakhya, L. Bingbing, Y. Rui, and W. Lin. A closed-form estimate of 3d ICP covariance. In 14th IAPR International Conference on Machine Vision Applications (MVA), pages 526-529, May 2015.

[16] S. I. Roumeliotis and G. A. Bekey. Distributed Multi-Robot Localization. In Lynne E. Parker, George Bekey, and Jacob Barhen, editors, Distributed Autonomous Robotic Systems 4, pages 179-188. Springer Japan, Tokyo, 2000.

[17] S. I. Roumeliotis and I. M. Rekleitis. Propagation of Uncertainty in Cooperative Multirobot Localization: Analysis and Experimental Results. Autonomous Robots, 17(1):41-54, July 2004.

[18] R. Smith, M. Self, and P. Cheeseman. Estimating Uncertain Spatial Relationships in Robotics. In Ingemar J. Cox and Gordon T. Wilfong, editors, Autonomous Robot Vehicles, pages 167-193. Springer, New York, NY, USA, 1990.

[19] S. Thrun, W. Burgard, and D. Fox. A real-time algorithm for mobile robot mapping with applications to multi-robot and $3 \mathrm{~d}$ mapping. In Proceedings ICRA. Millennium Conference. IEEE International Conference on Robotics and Automation. Symposia Proceedings (Cat. No.00CH37065), volume 1, pages 321-328 vol.1, April 2000.

[20] D. O. Wheeler, D. P. Koch, J. S. Jackson, T. W. McLain, and R. W. Beard. Relative Navigation: A Keyframe-Based Approach for Observable GPS-Degraded Navigation. IEEE Control Systems Magazine, 38(4):30-48, August 2018.

[21] Ph. Xu, G. Dherbomez, E. Héry, A. Abidli, and Ph. Bonnifait. System architecture of a driverless electric car in the grand cooperative driving challenge. IEEE Intelligent Transportation System Magazine, 10:4759, Spring 2018.

[22] X. Zhang, W. Xu, C. Dong, and J. M. Dolan. Efficient L-shape fitting for vehicle detection using laser scanners. In IEEE Intelligent Vehicles Symposium (IV), pages 54-59, June 2017. 